\title{
STAR CLUSTERS IN LOCAL GROUP GALAXIES
}

\author{
EDWARD W. OLSZEWSKI \\ Steward Observatory, University of Arizona \\ Tucson, AZ. 85721 USA \\ (edo@as.arizona.edu)
}

\section{Introduction}

In this paper I will try to give the flavor of two different concepts of stellar population, namely, the population of cluster systems within galaxies, and the stellar populations within individual clusters. I hope to show, in concert with other papers in this volume, that a generic vision of Population I and II has been outdated for a long time. I believe that it is still proper to talk about populations within individual objects; in the case of galaxies, one might make a correspondence of populations with the important star forming events in the history of a galaxy.

\section{The Decline of the Population Concept - Ages of Magellanic Cloud Clusters}

Shapley (1930) announced that there are a number of clusters in the Magellanic Clouds that look like globular star clusters, but that have A-type integrated spectra. Cannon had classified NGC 1872, 1903, 2041, 2107, 2134,2157 , and 2164 as A-type or A-?. To anticipate a later result, these clusters have SWB class (Searle et al., 1980) II-IV. With 15 years to go before Baade's seminal paper, the first evidence that populations were not universal, or at least were not simply two in number, had been found.

Shapely and Mohr (1932) used the LMC cluster system to help to delineate the size of the LMC. This particular paper claimed that NGC 1466 and NGC 2257, among others, were likely LMC "globular clusters," and concluded that these new clusters show that the LMC is $50 \%$ larger than expected from then-extant deep photographs. This idea, when combined with kinematics, has been important in deriving masses of galaxies (Olszewski et al., 1986; Zaritsky et al., 1989; Fich and Tremaine, 1991). Clus- 
ters are bright test particles, and can be seen, if they exist, even when the surface brightness of a galaxy has dropped to imperceptible levels.

Shapley and Nail (1951) announced the discovery of classical Cepheids (not RR Lyrae stars) within the cluster NGC 1866. Besides elucidating a major population difference between NGC 1866 and Galactic globular clusters, they also illustrated that the population of NGC 1866 was vastly different from that of the LMC field- NGC 1866 was $20 \times$ as efficient at making Cepheids as was the surrounding field. I will describe a more modern interpretation of this fact below.

Thackeray and Wesselink (1953) announced the long-awaited discovery of RR Lyrae stars in (or more often, about, see Olszewski, 1984, Fig. 2) one SMC and two LMC clusters. The inability to discover RR Lyraes in the LMC was partly caused by Shapley's incorrect distance determination, for the RR Lyraes were a full magnitude fainter than had been expected. Once again anticipating a problem that still plagues us, they pointed out that the uncertainty in the new LMC distance was dominated by photometry errors, but was also due to the "uncertainty in the assumed absolute magnitude of RR Lyrae variables."

Gascoigne and Kron (1952) measured colors of a number of Cloud clusters. To quote them, "the most striking feature is a strong tendency for a grouping of the colors into a blue group and a red group. The grouping appears so pronounced as to suggest that we have observed clusters of two distinct types."

In the late 1950 s through the early 1960 s, a number of workers, for instance Arp, Gascoigne, Hodge, Kron, and Tifft, made color-magnitude diagrams that showed that some Cloud clusters were significantly younger than the Galactic globulars. Arp $(1958 ; 1959)$ also showed that these clusters contain stars much redder than any found in Galactic globulars, that the SMC field seems to be made up of both old stars and young stars, and that the chemical composition of SMC cluster stars must be different from that of Galactic globular cluster stars. Again, to quote Arp, (1959) "regardless of the details of the differences, however, it is now necessary to abandon the hitherto useful working hypothesis that all galaxies contain the same kind of stars." Hodge (1963) and Gascoigne (1963) described how similar many color-magnitude diagrams of Cloud clusters were to those of intermediate-age Galactic open clusters.

Feast and Lloyd Evans (1973) showed that some of Arp's red stars were $\mathrm{C}$ stars, and advanced the hypothesis that perhaps C-star-containing clusters are younger than true globulars. They noted that there are $\mathrm{C}$ stars of similar absolute magnitudes in the Galactic open clusters NGC 7789, 2660, and 2477. Starting in the late 1970s, Aaronson and Mould (1982; 1985), Mould and Aaronson $(1979 ; 1980 ; 1982)$, Lloyd Evans (1980a; 1980b; 1983; 
1984) Frogel and Cohen (1982), and Frogel et al. (1990) collected observations that showed that the $\mathrm{C}$ and $\mathrm{M}$ stars in many of these clusters exceed the luminosity of the first-ascent giant branch tip, making them AGB stars. Mould and Aaronson (1980) derived cluster ages from the luminosity of the brightest $\mathrm{C}$ star, and noted that many clusters are significantly younger than 15 Gyr, including one cluster previously claimed to contain RR Lyraes (NGC 1978). Searle, Wilkinson, and Bagnuolo (1980) published integrated Gunn-system photometry of a large number of clusters, and proposed an age sequence. The large number of main-sequence color-magnitude diagrams now available have qualitatively confirmed both sets of ages, though the absolute ages implied from the earlier work needed adjustment, especially for clusters older than 1 Gyr. Similar, later work using integrated UBV photometry (Elson and Fall, 1985) has also had problems in getting the ages of the older clusters correct, and is generally not useful for deriving cluster formation rates.

It has been quite clear for a long time that cluster age distributions are quite different in each of the Magellanic Clouds, and in the Milky Way. If we lived in the LMC, how would the LMC-Baade have defined populations?

\section{LMC Clusters as a Stellar-Evolution Gold Mine}

It has been pointed out many times in the past that LMC clusters are a gold mine for understanding stellar evolution (see Robertson, 1973a,b, 1974; Hodge, and Flower, 1973; Flower and Hodge, 1975 for early examples of using modern evolutionary calculations to understand the ages of LMC clusters). A few other examples of the use of Magellanic Cloud clusters are the evolution of the luminosities of $\mathrm{C}$ and $\mathrm{M}$ stars (see above, but especially see Mould and Aaronson 1986; Frogel et al., 1990), theoretical studies of the clump giants (Seidel et al., 1987), observational studies of the magnitude of the clump (Olszewski et al., 1987; Hatzidimitriou 1991), evolution and formation of the red giant branch (Sweigart et al., 1990), evolutionary status and absolute magnitudes of RR Lyrae stars (Olszewski et al., 1987; Suntzeff et al., 1992; Walker 1992b), and use of convective overshoot in evolutionary models (Lattanzio et al., 1991). This list is necessarily incomplete. Many of these papers ultimately depend on the fact that absolute magnitudes of all stages of stellar evolution are quite precisely known, given the caveat that we know the distance to the LMC, because all LMC stars are at the same distance from the Sun to first order.

In 1987 we (see Mateo et al., 1990a,b; Olszewski et al., 1991; Welch et al., 1993a,b) began a systematic survey of the bluer LMC clusters with the intent of finding Cepheids. Data consist of time series of CCD images in several colors, using the Tololo $0.9 \mathrm{~m}, 4 \mathrm{~m}$, and the Las Campanas $1 \mathrm{~m}$ 
telescopes. In order to ensure some success, we started with the van den Bergh (1981) catalog of UBV colors of the clusters, and used fading models to scale the luminosity and color of each cluster to that of NGC 1866 . We then predicted the number of Cepheids expected, and observed a cluster if this predicted number exceeded 2 .

Our LMC-cluster Cepheid catalog has more than 50 members, counting the Cepheids in NGC 1866. Each cluster is in addition much more massive than Galactic open clusters and is generally lightly and evenly reddened, allowing a better understanding of the age and abundance of the cluster, and luminosity and colors of the Cepheids to be obtained. Most importantly, two clusters are now known with $15+$ Cepheids, and two more have a few Cepheids (see Table 1 Mateo et al., 1990b; and Table 1 in Welch et al., $1993 \mathrm{~b})$. While this is still work in progress, we can make several conclusions:

1. The PLC relation for cluster Cepheids is the same as that for field Cepheids. An important percentage of the short-period Cepheids pulsate in the first overtone mode. These Cepheids, of course, are easy to identify in the LMC simply by their offset in the PLC relation (see Fig. 2 in Olszewski et al., 1991). The older, photographic work on the LMC field does contain such stars; they typically were displaced in the PLC relation by the ratio of fundamental-mode to first-overtone periods, and commented upon in the notes to the table (for instance, in Fig. 1 of Martin et al., 1979). The overtone pulsators do typically have smaller amplitudes (and sinusoidal lightcurves), and may have been easily missed in the early Harvard surveys. Incidentally, these overtone pulsators are actually not rare in the field, as the spectacular PL relation of $\sim 1000$ Cepheids from the MACHO project (Cook et al., 1995) shows. We can test and refine the hypothesis of Bohm-Vitense $(1988,1994)$ that most short-period Cepheids pulsate in this mode. Clusters with blue loops that completely penetrate the instability strip seem to contain overtone pulsators; since the color of the blue loop is a function of age and metallicity you might expect the lower-metallicity LMC to have a higher percentage of overtone Cepheids than does the Milky Way.

2. When the blue loop is contained within the instability strip, we get many Cepheids. NGC 1866 and NGC 2031 are nice examples of this fact (see Fig. 1 of Olszewski et al., 1991 for the CMD of NGC 2031 with overlaid isochrones; and see Fig. 1 of Welch et al., 1993b for a CMD of NGC 1866). 3. There are nonvariables within the instability strip. This has been suggested for Milky Way field stars, but is difficult to prove (see Bidelman, 1985). High-resolution imaging is necessary to ensure that these nonvariables are not chance superpositions of stars.

4. The instability strip gets narower at lower luminosity, confirming the 
work of Fernie (1990) on Galactic Cepheids. The instability strip is clearly not bounded on the red and blue sides by parallel lines.

5. A single isochrone cannot explain the range in luminosity of Cepheids in a single cluster. There may still be some field-Cepheid contamination, which we can probably sort out with accurate velocities. There may also be Cepheids that have gained or lost mass because they are in close binary systems, a not uncommon occurrence (see Welch et al., 1991 for examples of both of these facts). We can also arbitrarily invoke differential mass loss, or higher crossings of the instability strip. A different, simple, testable hypothesis is that we are seeing a $20-30 \%$ age spread within these clusters. A spread of $2-3 \times 10^{7} \mathrm{y}$ would be as large or larger than larger than any spread convincingly verified in a cluster in the Milky Way. Prosser et al. (1994)argue that the stars in the Orion Trapezium cluster are $80 \%$ coeval to within $0.1 \times 10^{7} \mathrm{y}$, with $2 \%$ as old as $10^{7} \mathrm{y}$. They also point out that "the unambiguous detection of a range of formation times or 'age spread' amongst a cluster or association of stars would open up new avenues of study in the area of star formation research... Many attempts to make this type of measurement have been done before...but with somewhat mixed results." Eggen and Iben $(1988,1989)$ argue that there has been some star formation in many clusters on timescales larger than $10^{7} \mathrm{y}$. Other conflicting evidence can be given, but it seems clear that we do not yet know what age spread to "expect." It should be easier to derive the age spread in the LMC clusters because of the large numbers of stars, the identical distance to each star, and the low reddening to the typical cluster. Fig. 1 in Olszewski et al. (1991) shows Padova isochrones (a formal reference to these is Alongi et $a l ., 1993$; we used unpublished isochrones) fit to the CMDs of NGC 2031 and NGC 2136.

\section{Metallicities and Kinematics of the LMC Cluster System}

In the late 1970s and early 1980s it became possible to measure line strengths of individual red giants in Magellanic Cloud clusters (Cowley and Hartwick, 1982; Cohen, 1982) or to measure integrated colors and spectra (Smith et $a l ., 1988)$. Freeman, Ilingworth, and Oemler (1983) derived and compiled velocities of 59 clusters, and were able to make a first pass at deriving the kinematics of the cluster system as a function of SWB type (age). The most remarkable result was that the oldest clusters were in a planar distribution, not in a halo. Again, something about the LMC's oldest population is different from that of the Milky Way.

We have greatly improved our knowledge of the velocities and line strengths in approximately $80 \mathrm{LMC}$ clusters (Olszewski et al., 1991; Schommer et al., 1992; Suntzeff et al., 1992). A number of significant comments 
can be made from these papers:

1. The age-metallicity relation for clusters with good ages can be seen in Olszewski et al., (1991, Fig. 11). Aside from the cluster ESO121SC03, there are no clusters in the age range 2-3 to $12-15$ Gyr for the approximately 30 clusters that have well-determined ages; there is also a significant dearth of clusters in the abundance range -1.2 to -1.8 , even in the larger sample of 80 clusters (see Olszewski, 1993, Fig. 2). The age "gap" corresponds to an abundance "gap" as well. Either the LMC made no clusters during this time, or it only made clusters that could be destroyed in 2-3 Gyr. We have made crude arguments that the latter possibility is unlikely, though the weakest part of the argument is the number of giant molecular clouds in the LMC. There does seem to be corroborating evidence of such a period of little star formation from work on field stars (Bertelli et al., 1992), though their latest results (Vallenari et al., 1994) complicate the story.

2. We were able to show that the 13 clusters with ages like those of Galactic globulars do indeed have kinematics inconsistent with those expected for a halo (Freeman et al. were forced to use clusters as young as $\sim 2 \mathrm{Gyr}$ in their "oldest" sample. We find that the intermediate-aged LMC clusters are in the same disk as the youngest objects). Formally, if one excludes the old clusters superposed on the LMC bar, we get rotation solutions consistent with those derived for the youngest tracers, albeit with large errors. Hughes et al. (1991) have found a similar result for the oldest LPVs (see section 4.1.3 of Schommer et al., 1992). The system of OLPVs outside of $2 \mathrm{kpc}$ from the bar has large rotation and low $\mathrm{v} / \sigma$. Are there any populations in the LMC whose kinematics are consistent with those expected for a spherical halo? It seems unlikely given that the magnitude distribution of RR Lyraes suggests a flattened distribution (Walker, 1991). Isolating very metal poor giants and deriving their kinematics may be our last hope.

3. We were also able to compare the properties of the ensemble of old LMC clusters to various samples of halo Milky Way clusters. The most instructive sample of Milky Way clusters is that outside the solar circle. The mean absolute magnitude, standard deviation about that mean, distribution of RR Lyrae types, number of RR Lyrae stars per unit luminosity, and horizontal branch types (Walker, 1992a; see also Da Costa 1993) are the same for these two groups of galaxies. While van den Bergh (1994a,b) points out that the half-light radii of the two samples are different, my guess is that this property, of all the properties mentioned, is the most susceptible to environment. The sample of clusters outside the solar circle is "contaminated" with "young-halo" clusters; one or several proto-LMCs (not necessarily THE LMC) could have been swallowed up by the Milky Way to form the young halo.

We have now learned that the kinematics of the oldest clusters are 
different from those of the Milky Way, that these clusters most resemble the young-halo in the Milky Way, that the age-metallicity relation of the two galaxies is different, and that the distribution of ages of populous clusters is quite different. Is it really appropriate to say that any of the populations of clusters in the LMC is the same as populations of clusters in the Milky Way?

Hodge's (1989) population boxes provide much more information about the star formation histories and major distinguishing events than do the simple concepts of Population I and II. Population boxes are three dimensional diagrams with axes of star formation rate, time, and metallicity. While kinematics somehow needs to be added (and one could also envision adding $[0 / \mathrm{Fe}]$ from detailed abundances), by using population boxes we can at least admit similarities of star formation histories while accomodating important differences. We can imagine major events in the Milky Way during halo, disk, thick disk, and bulge formation, while simultaneously imagining an LMC which may have made few stars for several billions of years before "bursting."

\section{The Cluster Systems of M33 and M31}

I will not review in detail all that we know about the cluster systems in these two galaxies. Recent reviews ( Christian, 1993; Schommer, 1993; Huchra, 1993; Brodie, 1993) have nicely summarized the field. What I hope to do here is to point out some major features to buttress my argument that galaxies do have greatly differing populations. Schommer et al. (1991) point out that the M33 clusters have a range in colors, making this aspect of the M33 system more reminiscent of the LMC than of the Milky Way or M31. The bluer clusters follow disk rotation, the intermediate clusters have small (actually, indeterminate) rotation but a relatively small lineof-sight velocity dispersion, while the reddest clusters have no rotation, and a large line-of-sight velocity dispersion. M33 has a distinguishable old, spherical halo, making it different from the LMC. The gross details of the M33 cluster system are different from the details of both the LMC and of the Milky Way.

Two of the more obvious questions that one might ask about the M31 system are: how similar are the halo and disk globular cluster systems of M31 to those of the Milky Way; and is there a second parameter effect in the halo of M31 which would generalize the discovery of old halo and young halo in the Milky Way? A number of groups have discussed disk and halo systems in M31 (Ashman and Bird, 1993; Federici et al., 1993; Huchra et al., 1991; Elson and Walterbos, 1988), and there has been much recent work done on optical and infrared colors of M31 globulars (Reed et al., 1994; 
Cohen and Matthews, 1994; Fusi Pecci et al., 1993). Conclusions reached by the most recent papers are that M31 does indeed have both a disk and halo cluster system (Ashman and Bird), that there is little if any halo abundance gradient (Cohen and Matthews), and that the mean abundances of nuclear and outer clusters in M31 and the Milky Way are very similar (Cohen and Matthews; Ashman and Bird). These are not necessarily "new" conclusions (see Huchra et al., 1991, and references quoted above), but they have been confirmed or strengthened by new data and new analyses. Newer analyses will be strengthened by improvements in the fundamental data; more and better velocities and abundances will really help our comparison of the various cluster systems in nearby galaxies. Ashman and Bird further claim that the mean metallicities of any number of halo cluster systems are identical within the errors, which would have fundamental importance for galaxy formation. Some of the metallicity distributions seen in elliptical galaxies discussed in Bill Harris' review (this volume) make it unlikely that this last claim of Ashman and Bird is truly fundamental.

Within the next couple of years there will be color-magnitude diagrams of many M31 halo clusters. We will then know if the M31 cluster system has a second-parameter problem in its halo; if this turns out to be true then perhaps we can say that the M31 and Milky Way cluster populations are identical. Ajhar et al. (1994) showed color-magnitude diagrams of two M31 globulars, $\mathrm{K} 58$ and $\mathrm{K} 219$, with $[\mathrm{Fe} / \mathrm{H}]=-0.57$ and -1.83 , respectively. These WFPC2 data have a magnitude limit of $\sim 26.5$, giving well-delineated red-giant branches and horizontal branches. The CMD of K58 shows the red droop characteristic of a metal-rich population, and shows a stubby red horizontal branch. The RGB slope of K219 is much steeper, with a much bluer horizontal branch. The level of the horizontal branches is also different. As these and other data are published, we will improve our knowledge of the M31 halo and of the horizontal-branch magnitude as a function of metallicity.

\section{Other Studied Clusters in the Local Group}

The Fornax dwarf spheroidal galaxy also contains five globular clusters, several of which have been intensely studied (Buonanno et al., 1985, for instance). Some of these objects are also HST Cycle 4 targets. The CMDs of these objects resemble those of metal-poor clusters, though the HB morphologies are redder than expected for the metallicities. In the analyses of HB type and age versus metallicity (Zinn, 1993a; Chaboyer et al., 1992; van den Bergh, 1993a,b), the Fornax clusters fall neither on the old-halo line nor in the the young halo region. Zinn (1993b) argues that Fornax-like systems therefore could not form the halo. More massive, LMC-like systems 
are apparently needed. The newly-discovered Sgr galaxy has four globular clusters, and should help to refine this argument.

Olszewski and Aaronson (1985) discovered a density enhancement at or near the center of the Ursa Minor dwarf spheroidal galaxy, which we (Armandroff, Pryor and myself) have now confirmed with newer data (Irwin has also claimed to see a density enhancement at 1.5-2 core radii). If this "cluster" is at the center of Ursa Minor, it is dynamically stable. The CMD is indistinguishable from that of the field of UMi. With only 2 velocities of stars in this region, we know nothing about the dynamics of this feature. Could this feature be the most extreme "open" version of the nucleus of a nucleated dwarf? In the same vein, Seitzer and collaborators (private communication) have shown that the unresolved (in $1^{\prime \prime}$ seeing) central object in the Tucana system, which would have the colors and absolute magnitude of a globular if it were part of Tucana, is unresolved even with HST. It is therefore most likely a foreground star.

\section{Acknowledgments}

My work is partially supported by the NSF through grants AST $92-18030$ and AST 92-23967. I was also partially supported by a AAS International Travel Award. I thank Peter Strittmatter for additional support. I am grateful to Mario Mateo, Bob Schommer, and Nick Suntzeff for permission to discuss our work here, and to Ed Ajhar, Pat Seitzer, Gary Da Costa, Nick Suntzeff, and their collaborators for the privilege of showing their unpublished HST data at IAU 164. I acknowledge conversations with John Stauffer about age spreads in Galactic clusters, with Hugh Harris about details of the instability strip, and with Doug Welch about Cepheids in clusters.

\section{References}

Aaronson, M.A., and Mould, J.R., 1982, Ap. J. Suppl. 48, 161

Aaronson, M.A., and Mould, J.R., 1985, Ap. J. 288, 551

Ajhar, E. et al., 1994, Bull. A. A. S. 26, 938

Alongi, M., Bertelli, G., Bressan, A.,Chiosi, C., Fagotto, F., Greggio, L. and Nasi, E., 1993, A. \& A. Suppl. 97, 851

Arp, H., 1958, A. J. 63, 273

Arp, H., 1959, A. J. 64, 175

Ashman, K.M., and Bird, C.M., 1993, A. J. 106, 2281

Bertelli, G., Mateo, M., Chiosi, C., and Bressan, A., 1992, A. J. 103, 1859

Bidelman, W.P., 1985, Cepheids: Theory and Observations, ed. B.F. Madore, p.83

Bohm-Vitense, E., 1988, Ap. J. 324, L27

Bohm-Vitense, E., 1994, A. J. 107, 673

Brodie, J.P., 1993, The Globular Cluster-Galaxy Connection, ASP Conf. Series 48, eds. G.H. Smith and J.P. Brodie, p.483 
Buonanno, R., Corsi, C.E., Fusi Pecci, F., Hardy, E., and Zinn, R., 1985, A. \& A. 152, 65

Chaboyer, B., Sarajedini, A., and Demarque, P., 1992, Ap. J. 394, 515

Christian, C., 1993, The Globular Cluster-Galaxy Connection, ASP Conf. Series 48, eds. G.H. Smith and J.P. Brodie, p.448

Cook, K.H. et al., 1995, in proceedings of IAU Symposium 169.

Cowley, A.P., and Hartwick, F.D.A., 1982, Ap. J. 259, 89

Cohen, J.G., 1982, Ap. J. 258, 143

Cohen, J.G., and Matthews, K., 1994, A. J. 108, 128

Da Costa, G.S., 1993, The Globular Cluster-Galaxy Connection, ASP Conf. Series 48, eds. G.H. Smith and J.P. Brodie, p.363

Eggen, O.J., and Iben, I., Jr., 1988, A. J. 96, 635

Eggen, O.J., and Iben, I., Jr., 1989, $A . J$. 97, 431

Elson, R.A.W., and Fall, S.M., 1985, Ap. J. 299, 211

Elson, R.A.W., and Walterbos, R.A.M., 1988, Ap. J. 333, 594

Feast, M.W., and Lloyd Evans, T., 1973, M. N. R. A. S. 164, 16p

Federici, L., Bonoli, F., Ciotti, L., Fusi Pecci, F., Marano, B., Lipovetsky, V.A., Neizvestny, S.I., and Spassova, N., 1993, A. \& A. 274, 87

Fernie, J.D., 1990, Ap. J. 354, 295

Fich, M., and Tremaine, S., 1991, Ann. Rev. Astron. Astroph. 29, 409

Flower, P.J., and Hodge, P.W., 1975, Ap. J. 196, 369

Freeman, K.C., Illingworth, G., and Oemler, A., Jr., 1983, Ap. J. 272, 488

Frogel, J.A., and Cohen, J.G., 1982, Ap. J. 253, 580

Frogel, J.A., Mould, J., and Blanco, V.M., 1990, Ap. J. 352, 96

Fusi Pecci, F., Cacciari, C., Federici, L., and Pasquali, A., 1993, The Globular ClusterGalaxy Connection, ASP Conf. Series 48, eds. G.H. Smith and J.P. Brodie, p.410

Gascoigne, S.C.B., 1963, Observatory, 83, 71

Gascoigne, S.C.B., and Kron, G.E., 1952, P. A. S. P. 64, 196

Hatzidimitriou, D., 1991, M. N.R.A.S. 251, 545

Hodge, P.W., 1963, P. A. S. P. 75, 358

Hodge, P., 1989, Ann. Rev. Astron. Astroph. 27, 139

Hodge, P.W., and Flower, P.J., 1973, Ap. J. 185, 829

Huchra, J.P., 1993, The Globular Cluster-Galaxy Connection, ASP Conf. Series 48, eds. G.H. Smith and J.P. Brodie, p.420

Huchra, J., Brodie, J., and Kent, S., 1991, Ap. J. 370, 495

Hughes, S.M.G., Wood, P.R., and Reid, N., 1991, A. J. 101, 1304

Lattanzio, J.C., Vallenari, A., Bertelli, G., and Chiosi, C., 1991, A. $\mathscr{E} A$. 250, 340

Lloyd Evans, T., 1980a, M. N. R. A. S. 193, 87

Lloyd Evans, T., 1980b, M. N. R. A. S. 193, 97

Lloyd Evans, T., 1983, M. N. R. A. S. 204, 985

Lloyd Evans, T., 1984, M. N. R. A. S. 208, 447

Martin, W.L., Warren, P.R., and Feast, M.W., 1979, M. N. R. A. S. 188, 139

Mateo, M., Olszewski, E.W., and Madore, B.F., 1990a, Ap. J. 353, L11

Mateo, M., Olszewski, E.W., and Madore, B.F., 1990b, Confrontation Between Stellar Pulsation and Evolution, ASP Conf. Series 11, eds. C. Cacciari and G. Clementini, p.214

Mould, J., and Aaronson, M., 1979, Ap. J. 232, 421

Mould, J., and Aaronson, M., 1980, Ap. J. 240, 464

Mould, J., and Aaronson, M., 1982, Ap. J. 263, 629

Mould, J., and Aaronson, M., 1986, Ap. J. 303, 10

Olszewski, E.W., 1984, Ap. J. 284, 108

Olszewski, E.W., 1993, The Globular Cluster-Galaxy Connection, ASP Conf. Series 48, eds. G.H. Smith and J.P. Brodie, p.351

Olszewski, E.W., Mateo, M., and Madore, B.F., 1991, The Formation and Evolution of Star Clusters, ASP Conf. Series 13, ed. by K. Janes, p.588 
Olszewski, E.W., and Aaronson, M., 1985, A. J. 90, 2221

Olszewski, E.W., Peterson, R.C., and Aaronson, M., 1986, Ap. J. 302, L45

Olszewski, E.W., Schommer, R.A., and Aaronson, M., 1987, A. J. 93, 565

Olszewski, E.W., Schommer, R.A., Suntzeff, N.B., and Harris, H.C., 1991, A. J. 101, 515

Prosser, C.F., Stauffer, J.R., Hartmann, L., Soderblom, D.R., Jones, B.F., Werner, M.W., and McCaughrean, M.J., 1994, Ap. J. 421, 517

Reed, L.G., Harris, G.L.H., and Harris, W.E., 1994, A. J. 107, 555

Robertson, J.W., 1973a, Ap. J. 180, 425

Robertson, J.W., 1973b, Ap. J. 185, 817

Robertson, J.W., 1974, A. \& A. Suppl. 15, 261

Schommer, R.A., Olszewski, E.W., Suntzeff, N.B., and Harris, H.C., 1992, A. J. 103, 447

Schommer, R.A., 1993, The Globular Cluster-Galaxy Connection, ASP Conf. Series 48, eds. G.H. Smith and J.P. Brodie, p.458

Schommer, R.A., Christian, C.A., Caldwell, N., Bothun, G.D., and Huchra, J., 1991, A. J. 101, 873

Searle, L., Wilkinson, A., and Bagnuolo, W. G., 1980, Ap. J. 239, 803

Seidel, E., Da Costa, G.S., and Demarque, P., 1987, Ap. J. 313, 192

Shapley, H., 1930, Star Clusters, McGraw-Hill: New York

Shapley, H., and Mohr, J., 1932, Harv. Obs. Bull. 889, 13

Shapley, H., and Nail, V.M., 1951, A. J. 55, 249

Smith, H.A., Searle, L., and Manduca, A., 1988, Globular Cluster Systems in Galaxies, IAU Symp. 126, eds. A.G.D. Philip and J.E. Grindlay, p.563

Suntzeff, N.B., Schommer, R.A., Olszewski, E.W., and Walker, A.R., 1992, A. J. 104, 1743

Sweigart, A.V., Greggio, L., and Renzini, A., 1990, Ap. J. 364, 527

Thackeray, A.D., and Wesselink, A.J., 1953, Nature, 171, 693

Va!lenari, A., Bertelli, G., Chiosi, C., and Ortolano, S., 1994, ESO Messenger, 76, 30

Van den Bergh, S., 1981, A. \& A. Suppl. 46, 79

Van den Bergh, S., 1994a, preprint

Van den Bergh, S., 1994b, preprint

Van den Bergh, S., 1993a, A. J. 105, 971

Van den Bergh, S., 1993b, Ap. J. 411, 178

Walker, A.R., 1991, The Magellanic Clouds, IAU Symposium 148, eds. R. Haynes and D. Milne, p.307

Walker, A.R., 1992a, A. J. 104, 1395

Walker, A.R., 1992b, Ap. J. 390, L81

Welch, D.L., Mateo, M., Côté, P., Fischer, P., and Madore, B.F., 1991, A. J. 101, 490

Welch, D.L., Mateo, M., Olszewski, E.W., Fischer, P., and Takamiya, M., 1993a, A. J. 105, 146

Welch, D.L., Mateo, M., and Olszewski, E.W., 1993b, New Perspectives on Stellar Pulsation and Pulsating Variable Stars, IAU Colloq. 139, eds. J.M. Nemec and J.M. Matthews, p.359

Zaritsky, D., Olszewski, E.W., Schommer, R.A., Peterson, R.C., and Aaronson, M., 1989, Ap. J. 345, 759

Zinn, R., 1993a, The Globular Cluster-Galaxy Connection, ASP Conf. Series 48, eds. G.H. Smith and J.P. Brodie, p.38

Zinn, R., 1993b, The Globular Cluster-Galaxy Connection, ASP Conf. Series 48, eds. G.H. Smith and J.P. Brodie, p.302

HARRIS: I'd like to support the view that both you and Mike Feast have hinted at strongly, which is that there are no particular things called "globular" and "open" clusters; there are only star clusters, at all ages and metallicities. The major thing you need to form a really massive (=globular) star cluster is just to accumulate a large enough supply of gas in a giant molecular cloud, and it doesn't really matter 
when in a galaxy's history that happens. In some sense, it was obviously easier for the LMC and M33 to do that over a longer period of time than it was for the Milke Way or M87.

FEAST: I was interested in your remark that the ratio of numbers of Cepheids pulsating in the fundamental and the first overtone depends strongly on the evolutionary loops in the HR diagram. One might perhaps then expect that the ratio would be rather sensitive to metallicity. In fact, it seems that the number ratio increases rapidly at about the same period in both the SMC and the LMC (overtones frequent for period less than about 10 days), suggesting rather little metallicity dependence.

OLSZEWSKI: I think that one can only learn the answer to your comments from studying Cpeheids in clusters, where one can hope to isolate age and abundance. TAYLER: I do not understand why you were surprised by an apparent age spread of $10^{7}$ years in a cluster. What is there to stop star formation other then the explosion of the first supernova, which takes that long? It is true that to a close approximation in old clusters stars have the same age, but not in very young clusters where the age spread can be larger than the age.

OLSZEWSKI: I was not speaking of VERY young clusters. I provide additional evidence about age spreads in the text. I was also speaking of empirical evidence, not about expectations.

BRODIE: I strongly disagree with your statement that new IR colors will improve the metallicity estimates for M31 globulars. Estimates based on spectroscopic line strength indices (especially those which include a number of different elements line strengths, as is the case for the Huchra, Brodie and Kent metallicity determinations) are inherently more reliable than those based on colors. The HBK metallicities each carry error estimates which accumulately reflect the $\mathrm{S} / \mathrm{N}$ of the individual spectra (as well as other uncertainties). You mentioned that Cohen's IR color-based metallicity estimates differ from the HBK spectroscopic estimates for a few halo metal-rich clusters. It does not follow that the IR estimates are the correct ones and that the HBK ones should be "thrown out", as you stated in the talk. In general, though, the agreement is excellent and we derive identical mean metallicities for the system as a whole.

OLSZEWSKI: What I said in my talk must have been too strong (though I cannot remember the exact words I said). All I intended was that the fundamental data can use improvement, especially in the case of clusters with quoted large metallicity errors. Some of this improvement can come about with IR photometry, but improved spectroscopy could make the same improvements. As non-observers use these catalogs without the same appreciation of the errors as observers, one must be careful about publishing data with large error bars. Error bars are easily forgotten in a clever mathematical analysis by someone years down the road. 